\title{
Rats in a levered T-maze task show evidence of time-place discriminations in two different measures
}

\author{
Scott H. Deibel ${ }^{1}$ - Andrew B. Lehr ${ }^{1}$ - Chelsea Maloney ${ }^{1} \cdot$ Matthew L. Ingram $^{1}$ • \\ Leanna M. Lewis ${ }^{1}$ - Anne-Marie P. Chaulk ${ }^{1}$ Pam D. Chaulk ${ }^{1}$ - Darlene M. Skinner ${ }^{1}$. \\ Christina M. Thorpe ${ }^{1}$
}

Published online: 7 December 2016

(C) Psychonomic Society, Inc. 2016

\begin{abstract}
It is difficult for rats to learn to go to an arm of a T-maze to receive food that is dependent on the time of day, unless the amount of food in each daily session is different. In the same task, rats show evidence of timeplace discriminations if they are required to press levers in the arms of the T-maze, but learning is only evident when the first lever press is considered, and not the first arm visited. These data suggest that rats struggle to use time as a discriminative stimulus unless the rewards/events differ in some dimension, or unless the goal locations can be visited prior to making a response. If both of these conditions are met in the same task, it might be possible to compare time-place learning in two different measures that essentially indicate performance before and after entering the arms of the T-maze. In the present study, we investigated time-place learning in rats with a levered Tmaze task in which the amounts of food varied depending on the time of day. The first arm choices and first lever presses both indicated that the rats had acquired timeplace discriminations, and both of these measures became significantly different from chance during the same block. However, there were subtle differences between the two measures, which suggest that time-place discrimination is aided by visiting the goal locations.
\end{abstract}

Keywords Time-place learning $\cdot$ Time-place discrimination · Daily $\cdot$ Circadian $\cdot$ Differential reinforcement

Christina M. Thorpe

cthorpe@mun.ca

1 Department of Psychology, Memorial University of Newfoundland, St. John's, Newfoundland, Canada A1B 3X9
For the past 30 years, psychologists and biologists have studied how animals learn about events that occur in different locations at different times of day. This ability is known as daily time-place learning or time-place discrimination, and has been observed in both the field (Daan \& Koene, 1981; Wilkie et al., 1996) and the lab (Biebach, Gordijn, \& Krebs, 1989; Saksida \& Wilkie, 1994). Gallistel (1990) suggested that animals automatically encode the time and place of biologically significant events.

In rats, although many studies have observed evidence of daily time-place learning (Aragona, Curtis, Davidson, Wang, \& Stephan, 2002; Carr \& Wilkie, 1997b; Deibel \& Thorpe, 2013; Lukoyanov, Pereira, Mesquita, \& Andrade, 2002; Mistlberger, De Groot, Bossert, \& Marchant, 1996; Pizzo \& Crystal, 2002, 2004b, 2006; Van der Zee et al., 2008; Widman, Gordon, \& Timberlake, 2000; Widman, Sermania, \& Genismore, 2004), some have only done so by increasing the cost of making an error (response cost), or by altering the events associated with each daily session (Aragona et al., 2002; Boulos \& Logothetis, 1990; Lukoyanov et al., 2002; Means, Arolfo, Ginn, Pence, \& Watson, 2000; Thorpe, Bates, \& Wilkie, 2003; Thorpe \& Wilkie, 2007; Widman et al., 2000; Widman et al., 2004). Contrary to Gallistel's (1990) theory, it appears that rats might not readily associate time, place, and event information codes in all relevant situations. Alternatively, maybe task selection or other variables are masking evidence of daily time-place learning. In this article, we explore how changing the events associated with each daily session might affect rats' ability to learn a daily time-place learning task as assessed by two different performance measures.

In a series of experiments, Means and colleagues (Means, Arolfo, et al., 2000; Means, Ginn, Arolfo, \& Pence, 2000) found that rats had great difficulty with going to one arm of a T-maze in the morning and the other arm in the afternoon for 
food rewards. Thorpe and colleagues (2003) extended this finding to a variety of aversive and appetitive tasks. Means and colleagues (Means, Arolfo, et al., 2000) had suggested that rats cannot use time of day as a discriminative stimulus (i.e., as "a signal for a specific response," p. 13) while in the start arm of a T-maze. Instead, they posited that time of day is an occasionsetter, which simply indicates whether food is available. Once in the correct location (i.e., at the end of the choice arms where the levers are located), responding is guided by local cues (Means, Arolfo, et al., 2000; Means, Ginn, et al., 2000).

The idea that time acts as an occasion-setter is bolstered by the finding that rats can acquire time-place discriminations in a go-no-go T-maze task, in which one of the daily sessions is associated with food and the other session is not (Means, Arolfo, et al., 2000; Thorpe et al., 2003). An occasion-setter could facilitate time-place discriminations in this case because food is available only in one session. Rats can also acquire time-place discriminations in the T-maze when they are required to press levers located at the ends of arms for food in each daily session (Deibel \& Thorpe, 2013; Mistlberger et al., 1996). However, learning is only evidenced when the first lever pressed is considered, and not the first arm entered (Deibel \& Thorpe, 2013; Mistlberger et al., 1996). As was suggested by Means and colleagues (Means, Arolfo, et al., 2000; Means, Ginn, et al., 2000), it is possible that when they are in the start arm, rats are only aware that food is available, and thus fail to learn to go to the correct arm first. However, when in the goal locations, local cues signal which lever to press, depending on the time of day.

We recently demonstrated that rats did not show time-place learning in an operant box when the same lever was used in two daily sessions that had different fixed-interval schedules (Deibel et al., 2014). However, when each daily session was associated with a different lever, the rats showed evidence of time-place learning by responding more in the beginning of the session with the shorter fixed-interval schedule (Deibel et al., 2014). These data also support the notion that local cues specific to the goal locations are needed for rats to show evidence of time-place discriminations.

Along the same vein as the go-no-go task, rats acquired a T-maze time-place learning task when the amounts of food were different in the two daily sessions (Thorpe \& Wilkie, 2007). In contrast to the T-maze version with equal amounts of food, by varying the amounts of food available in the two daily sessions, two distinct events are thought to be created (Thorpe \& Wilkie, 2007). The development of correct firstarm-choice performance suggested that in this case the rats could use time as a discriminative stimulus before entering the arms of the T-maze.

In summary, in the studies discussed above, it appears that rats have trouble using time as a discriminative stimulus unless the events associated with each daily session differ in some other dimension, the response cost is increased, or the task parameters allow the rats to sample the goal locations before executing a response. As we mentioned above, the first arm choice only suggests evidence of time-place discriminations in the nonlevered T-maze when the events associated with each daily session differ in some other dimension, whereas lever pressing and not the first arm choice indicates evidence of time-place discriminations in a T-maze with levers at the end of each arm. Thus, if the amounts of food delivered by lever presses differ in each daily session, might the first arm choice and first lever presses both show evidence of timeplace discriminations? If so, would both measures show evidence of time-place discrimination equally and at the same block in the experiment? The present study investigated rats' ability to acquire a levered T-maze task in which one lever provided a large amount of food in morning sessions and the other lever provided a small amount of food in afternoon sessions. We hypothesized that rats' first press and first arm choice would show evidence of time-place learning, and that the first arm choice would take longer to acquire and be less robust than the first lever press.

\section{Method}

\section{Subjects and apparatus}

Thirteen male Long-Evans rats, weighing an average of $180 \mathrm{~g}$ at the start of training, were purchased from Charles River (St. Constant, Quebec). The rats were fed a standard daily diet of Laboratory Animal Feed (PMI Nutrition International, St. Louis, MO) so that their weights were maintained at approximately $85 \%$ of their free-feeding weights, while being allowed to gain approximately $5 \mathrm{~g}$ per week to account for growth. This level of deprivation was not considered extreme, since the animals remained healthy when this feeding regime was used in our previous studies. Rats were given free access to water, except during the experimental sessions. The guidelines outlined by the Canadian Council on Animal Care were followed.

The rats were individually housed in transparent plastic cages $(45 \times 25 \times 21 \mathrm{~cm})$ lined with corncob bedding (Necto Company, New York, NY). The cages also contained shredded paper for nesting and a wooden block and Nylabone (Nylabone Products, Neptune, NJ) for enrichment. A 12:12 h light:dark cycle was used in the colony room, with lights on at $0700 \mathrm{~h}$ and off at $1900 \mathrm{~h}$.

The experimental apparatus described in Deibel and Thorpe (2013) was used in the present study. Briefly, an open T-maze with start and choice arms $15 \mathrm{~cm}$ wide and $53 \mathrm{~cm}$ long was elevated $85 \mathrm{~cm}$ above the floor. At the end of each of the choice arms, a 5-cm nonretractable lever (Model ENV-110 M, Med Associates, Inc., St. Albans, Vermont, USA), 4-cm-wide food cup, light, and pellet dispenser (Model ENV-203045, 
Med Associates, Inc., St. Albans, Vermont, USA) were mounted on a Plexiglas wall. The lever was always positioned on the side closest to the start arm, with the food cup next to it and the light centered above the lever and food cup. This meant that in the left choice arm, the lever was to the left of the food cup, whereas in the right choice arm, the lever was to the right of the food cup. Each lever, food cup, light, and pellet dispenser was positioned $8,6,14$, and $40 \mathrm{~cm}$ above the maze, respectively. The experimental room $(604 \mathrm{~cm} \times 248 \mathrm{~cm})$ contained a variety of extramaze cues, such as a computer desk, a stool with a radio, an air conditioner, and a countertop. A computer, with in-house-written Python software, was located in a room just outside the experiment room and was used to run and collect all of the data for the experiment.

\section{Procedure}

Prior to the start of the experiment, the rats were pretrained to lever press in a Plexiglas operant conditioning box $(47 \times 47 \times$ $32 \mathrm{~cm}$ ) that contained a retractable lever (Med Associates Inc., St. Albans, Vermont) in the center of each of its four walls. Pretraining occurred in an operant conditioning box, and rats were shaped for 18 days until all were consistently pressing on a variable-ratio (VR) 30 schedule. The rats were then habituated to the T-maze for two days, during which both levers provided reinforcement on a VR 30 schedule. Pretraining and habituation occurred at times other than those used for the experimental sessions (i.e., in the middle of the day). For all pretraining and experimental sessions, 45-mg Dustless Precision Pellets (BioServ, Frenchtown, NJ) were used for reinforcement.

For each rat, the experimental trials were run twice daily five days per week. Morning sessions ran from approximately $0830 \mathrm{~h}$ to $0930 \mathrm{~h}$, and afternoon sessions ran from approximately $1430 \mathrm{~h}$ to $1530 \mathrm{~h}$. The rats were tested in the same order during all sessions. One lever provided reinforcement in the morning sessions, and the other lever provided reinforcement in the afternoon sessions. The correct lever always provided reinforcement according to a VR 5 schedule. However, in one of the two daily sessions, the rats received only one pellet (small reinforcer) for each reinforcement, whereas in the other daily session, each reinforcement consisted of five pellets (large reinforcer). The arm locations and amount of reinforcement were constant throughout training for each rat (e.g., Rat 1 always received a small reinforcer on Arm 1 in morning sessions and a large reinforcer on Arm 2 in afternoon sessions). However, these two variables were counterbalanced across rats.

Each session lasted a total of $240 \mathrm{~s}$. At the start of each session, rats were placed on the T-maze at the base of the start arm. The lights on both choice arms turned on, and the reward immediately became available at the correct lever. The rats were trained for 80 days.
The computer program recorded the time and location of each press and whether that press elicited a reinforcer. The dependent measures consisted of the percentages of correct first presses and first arm choices.

To determine the type of timer used, rats were given four or five skip-session probes (at least two morning and two afternoon). The probe trials were conducted after the end of the last training block ( Day 82$)$, so that these skipped sessions would not confound the learning during training. For these probes, rats were excluded from either a morning or an afternoon session (i.e., the rat would remain in the colony room for that session), and first lever presses were noted in the following session. Each rat was given these probe sessions in a semirandomized order. After a rat had completed its first probe, a subsequent probe was not administered until the rat had made $4 / 4$ or $5 / 6$ correct first presses in the normal experimental trials. Probe sessions were only administered if the rats had been tested on the previous day and were going to be tested on the following day. After the four skip probe trials, the rats that got one correct and one incorrect session in either the skip AM or the skip PM probe trials were given another skip probe trial for that particular session (see Table 2 below).

In circadian timing, different phase angles of an oscillator are associated with the two daily events (Carr \& Wilkie, 1997a; Deibel \& Thorpe, 2013). Thus, rats were deemed to be using a circadian timer if they generally responded correctly following a skip probe, regardless of which session was skipped. In ordinal timing, rats learn the order of the two daily events (trial order), but not the absolute times of those events (Carr \& Wilkie, 1997a; Deibel \& Thorpe, 2013). Rats were deemed to be using an ordinal timer if they generally responded incorrectly following skip AM sessions and correctly after skip PM sessions. Finally, in nontiming alternation, rats learn to go to the location that was not visited in the previous daily session (Carr \& Wilkie, 1997a). Thus, rats were classified as using nontiming alternation if they generally responded incorrectly following both skip AM and skip PM sessions.

Following the skip probes ( $\sim-2$ days after), the rats received training for 15 more days, except that the amounts of reinforcers were now equal in both daily sessions. For half of the rats, the amount of food on these equal sessions was the small amount (i.e., one pellet). For the other half, the amount of food was the large amount (i.e., five pellets).

\section{Statistics}

Data were only included in the analyses if the animals had been tested in both of the daily sessions. Day 9 was excluded from the analyses for all rats, because there was a computer malfunction and data were collected in only one of the daily sessions. Additionally, this occurred for one rat on the first day of testing. 
The 80 days of training were divided into eight blocks of ten days. Separate repeated measures analyses of variance (ANOVAs) were used to assess learning in each measure across the duration of the differential amounts of training. Although ANOVAs were used to analyze performance across experimental blocks, since our a priori hypothesis was concerned with when the rats acquired the task, the performance in each block was compared to chance $(50 \%)$. Rats were considered to have learned the task if they reached a criterion of 18/20 correct trials. Accuracy was calculated for first presses and first arm choices.

For the final blocks of equal-amounts training, the 15 days were divided in three blocks of five days. The same measures and statistics were used as previously, except that because we were interested in determining whether the change in schedule caused a decrement in performance in both first presses and first arm choices, we used paired-samples $t$ tests to compare the last ten days of differential-amounts training (Block 8) to the first ten days of equal-amounts training (Blocks 1 and 2). One rat was removed from these analyses because it did not receive the full 15 days of training on this schedule.

For the ANOVAs and paired-samples $t$ tests used to assess the equal-amounts training, alpha was set at .05. However, because many one-sample $t$ tests were used to determine whether the performance in every block was statistically different from chance for each measure type, alpha was reduced to .01 to reduce the probability of Type I error.

\section{Results}

\section{Differential amounts of food training}

As is shown in Fig. 1a and $\mathrm{b}$, for both first presses $[F(7,84)=$ $35.09, p<.01]$ and first arm choices $[F(7,84)=7.892, p<$

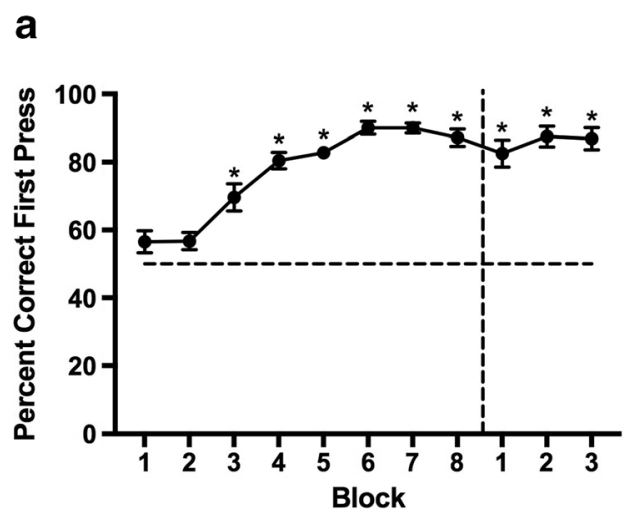

Fig. 1 First press and first arm choice performance. (a) Percent correct first presses during the eight blocks of differential-amounts training ( $n=$ 13 ) and the three blocks of equal-amounts training $(n=12)$. (b) Percent correct first arm choice performance during the eight blocks of differential-amounts training $(n=13)$ and the three blocks of equalamounts training $(n=12)$. The horizontal dotted line represents chance
.01], main effects of block emerged. First presses were significantly above chance in every block except the first two (see also Table 1). The first arm choices were significantly different from chance in all blocks except for Blocks 1, 2, and 4. All of the rats achieved a criterion of 18 correct first presses in 20 trials, and they did so on average early in Block 5 (42.23 days). Only four rats achieved a criterion of 18 correct first arm choices in 20 trials during the experiment, and on average they did so in Block 6 (55.38 days).

\section{Skip-session probes}

Eight of the 13 rats appeared to be using a circadian timer, since they tended to respond correctly (first press) in both sessions following the skipped sessions. One rat was alternating, two rats were using ordinal timers, and the remaining two rats' behavior was unclassifiable, which appeared to be a mix of both alternating and circadian timing (see Table 2).

\section{Equal amounts of food training}

As is depicted in Fig. 1a, no main effects of block emerged for either first presses or first arm choices during equal-amounts training. Correct first presses were significantly above chance in each block (see Table 1), and performance was not significantly different when the last ten days of differential-amounts training were compared to the first ten days of equal-amounts training. For correct first arm choices, performance was only significantly above chance in the third block (see Fig. 1b and Table 1), and the percentage of correct first arm choices in the first ten days of equal-amounts training was significantly less than in the last ten days of differentialamounts training $[t(11)=2.39, p=.04]$.

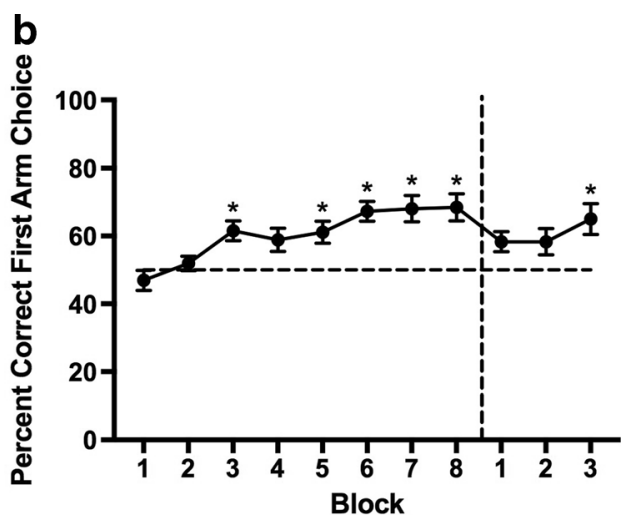

(50\%), and the vertical dotted line indicates when equal-amounts training started. The blocks before the vertical dotted line consist of 10 days, whereas the blocks after consist of five days. Error bars represent the standard errors of the means, and an * indicates that performance for that block was statistically different $(p<.01)$ from chance 
Table 1 Mean percent correct first presses and first arm choices, with the accompanying results from the one-sample $t$ tests

\begin{tabular}{llllllll}
\hline & \multicolumn{2}{l}{ First Presses } & & & \multicolumn{2}{l}{ First Arm Choices } \\
\cline { 2 - 3 } \cline { 6 - 7 } Block & $M$ & $t$ & $p$ & & $M$ & $t$ & $p$ \\
\hline 1 & 56.54 & 2.04 & .06 & & 46.92 & -1.04 & .32 \\
2 & 56.74 & 2.67 & .02 & 51.92 & 0.89 & .39 \\
3 & 69.62 & 4.88 & $<.01^{*}$ & 61.54 & 3.97 & $<.01^{*}$ \\
4 & 80.45 & 12.55 & $<.01^{*}$ & 58.87 & 2.60 & .02 \\
5 & 82.77 & 19.82 & $<.01^{*}$ & 61.15 & 3.42 & $.01^{*}$ \\
6 & 90.06 & 21.46 & $<.01^{*}$ & 67.31 & 5.93 & $<.01^{*}$ \\
7 & 90.00 & 26.71 & $<.01^{*}$ & 68.08 & 4.68 & $<.01^{*}$ \\
8 & 87.18 & 14.60 & $<.01^{*}$ & 68.46 & 4.64 & $<.01^{*}$ \\
1 & 82.50 & 8.30 & $<.01^{*}$ & 58.33 & 2.80 & .02 \\
2 & 87.50 & 12.31 & $<.01^{*}$ & 58.33 & 2.16 & .05 \\
3 & 86.89 & 11.40 & $<.01^{*}$ & 65.00 & 3.32 & $<.01^{*}$ \\
\hline
\end{tabular}

$M$ represents the mean percent correct performance in each block, and $t$ and $p$ represent the $t$ and $p$ values of one-sample $t$ tests, respectively. For example, in Block 1, the mean percent correct first presses was 56.54, which was not significantly different from chance $[t(12)=2.04, p=.064]$. Significance was set at $p<.01$ and is indicated by an asterisk. In one instance, a $p$ value of .01 is considered significant because it was less than .01 and was rounded up. The horizontal line after Block 8 represents when equal-amounts training started. The block size for the eight blocks of differential-amounts training $(n=13)$ was ten days, whereas the block size for equal-amounts training $(n=12)$ was five days

\section{Discussion}

In the present study, we sought to determine whether firstarm-choice performance in a levered T-maze task would show evidence of learning if the amounts of food were different in the two daily sessions. As expected, the rats' first-arm-choice

Table 2 Probe trial performance during the skip-session probe trials

\begin{tabular}{llll}
\hline Rat & Skipped AM & Skipped PM & Strategy \\
\hline 1 & ICI & II & alternation \\
2 & CC & CIC & circadian \\
3 & CII & CC & ordinal \\
4 & CC & II & other \\
5 & ICC & IC & other \\
6 & CC & CC & circadian \\
7 & CIC & CC & circadian \\
8 & CC & CC & circadian \\
9 & CIC & CC & circadian \\
10 & CC & CC & circadian \\
11 & CC & CC & circadian \\
12 & CC & CC & circadian \\
13 & ICI & CC & ordinal \\
\hline
\end{tabular}

I and $\mathrm{C}$ indicate that the rat's first press in the subsequent session after the skipped one was incorrect and correct, respectively. Within each column, each letter represents performance in one skip probe trial and first-lever-pressing behavior suggested that the rats had acquired time-place discriminations. Interestingly, performance in both measures was first statistically different from chance in Block 3. When the amounts of food delivered in each session were made equal, there was a decrement in percent correct first-arm-choice performance. Although firstpress performance was unaffected, it remains a possibility that the decrement in first-arm-choice performance was due to the reward amount conflicting with the previously learned information.

Previous data had suggested that rats do not acquire timeplace discriminations unless the response cost is high (Lukoyanov et al., 2002; Widman et al., 2000; Widman et al., 2004), the daily events differ in some way other than time of day (Means, Arolfo, et al., 2000; Thorpe et al., 2003; Thorpe \& Wilkie, 2007), or the rats are allowed to explore the goal locations before executing a response (Deibel \& Thorpe, 2013; Mistlberger et al., 1996; Pizzo \& Crystal, 2002, 2004a, 2006). The idea is that unless these conditions are met, time can not be used as an discriminative stimulus, but instead acts as an occasion-setter (Means, Arolfo, et al., 2000; Means, Ginn, et al., 2000). Interestingly, in situations such as a levered T-maze task in which rats are allowed to explore the goal locations before making a response, evidence of learning is only shown by the first lever pressed, and not by the first arm visited (Deibel \& Thorpe, 2013; Mistlberger et al., 1996). One interpretation is that before a rat enters the arms of the T-maze, time acts as an occasion-setter that does not provide information as to which arm contains the reward. When in the goal location, it has been suggested that local cues facilitate timeplace discrimination and guide lever responding. The finding that time does not act as a discriminative stimulus when rats respond at the same place at two different times of day, but does when each daily session is associated with distinct spatial locations, particularly supports the local-cue argument (Deibel et al., 2014).

An alternative explanation for the lack of first arm choices in the T-maze tasks is that while in the start arm, the rats do know the location of the food reward, but for some reason they do not choose the correct arm, perhaps because of some species-typical behavior associated with exploring the maze. However, if this were the case, then one would not expect that having different amounts of food available would decrease this tendency. Instead, it seems more likely that when in the start arm for the same event, rats cannot use time as a discriminative stimulus.

The data from the present study fit with the finding that rats' first arm choices show evidence of time-place discriminations in a nonlevered T-maze with differential amounts of food (Thorpe \& Wilkie, 2007). As in the nonlevered T-maze version of this task (Thorpe \& Wilkie, 2007), the rats in the present study were predominantly using a circadian timing strategy to solve the task. 
The finding that both first-arm-choice and first-press performance measures indicated learning with different reward amounts suggests that similar processes were at play. However, despite both measures showing evidence of learning, we observed some subtle differences between them, which suggests that the first arm choice was not as robust as the first lever press. For example, only four of the 13 rats achieved a criterion of 18 correct first arm choices out of 20 , and it took these rats longer to achieve this than the average days required to reach criterion for first presses. The percentage of correct first presses was also much higher than that for first arm choices for the entire duration of the experiment. Finally, percent correct first presses remained statistically significantly different from chance and did not decrease after the switch to equal-amounts-of-food training, whereas first-armchoice performance was attenuated and not significantly different from chance during the first ten days of this schedule.

In the present study, the fact that a correct first arm choice appears harder for the rats to execute than a correct first press, even with differing food amounts, suggests that different processes may underlie decision making before and after entering the arms of the T-maze. First-arm-choice behavior suggests that the rats are able to use time as a discriminative stimulus before entering the arms of the T-maze; however, evidence of time-place discriminations is more robust when the rats can visit the goal locations.

As we discussed above, it is likely that local cues associated with the goal locations allow time and place information to better guide responding. The levered T-maze task differs from its nonlevered counterpart in a variety of ways that might promote the use of local cues. First, the locations of the reinforcers are likely more salient in levered tasks because the rats spend more time per trial in the goal location (e.g., minutes), as compared to only a few seconds in the discrete-trials version. Also, as we discussed above, without being scored as an error, the operant nature of the task allows the rats to explore both goal locations before they make a lever press. As a result, it might be easier for the rats in the levered version of the task to distinguish the two different goal locations. Also, in each session the rats received a larger amount of food in the levered version (on average, 78.35 45-mg reinforcers in the large reinforcer session, and 29.01 45-mg reinforcers in the small reinforcer session) of the task than in the discrete-trial version of the task (one Froot Loop in the large reinforcer session and 1/5 of a Froot Loop in the small reinforcer sessions).

In conclusion, altering the reward/event on the dimension of quantity is enough to produce evidence of time-place discrimination before entering the goal locations, which is not normally seen in nonlevered (Means, Arolfo, et al., 2000; Means, Ginn, et al., 2000; Thorpe et al., 2003) and levered (Deibel \& Thorpe, 2013; Mistlberger et al., 1996) T-maze tasks when the reward/events are the same. Despite our (Deibel \& Thorpe, 2013) and other labs' (Mistlberger, De
Groot, Bossert, \& Marchant, 1996) failure to observe firstarm-choice learning in levered T-maze tasks with equal amounts of food in both daily sessions, it should be noted that in the present study - because we did not have a control group that received the same amount of food in both daily sessions the first-arm-choice behavior could have been due to procedural differences rather than the different reward amounts. Nonetheless, taken together with the differential food amounts in the nonlevered T-maze (Thorpe \& Wilkie, 2007), we have added the lever dimension and found that in the same task there are subtle differences between the first arm choice and the first lever press, though the patterns of results are the same. With these data, we are homing in on the conditions under which time can act as a discriminative stimulus.

Author note This research was supported by the Natural Sciences and Engineering Research Council of Canada (NSERC). A special thank you to Darcy Hallett for statistical consulting, and also to Robert J. McDonald for reading a version of the manuscript. We also thank Stephanie Bennett, Courtney Clarke, Isaac Hughes, and Hiliary Martin for assistance running the experiments and doing data entry. S.H.D. currently holds an NSERC CREATE BIP PhD scholarship and is now at the Canadian Centre for Behavioural Neuroscience, University of Lethbridge.

\section{References}

Aragona, B. J., Curtis, J. T., Davidson, A. J., Wang, Z., \& Stephan, F. K. (2002). Behavioral and neurochemical investigation of circadian time-place learning in the rat. Journal of Biological Rhythms, 17, 330-344. doi:10.1177/074873002129002636

Biebach, H., Gordijn, M., \& Krebs, J. (1989). Time-place learning by garden warblers, Sylvia borin. Animal Behaviour, 37, 353-360.

Boulos, Z., \& Logothetis, D. E. (1990). Rats anticipate and discriminate between two daily feeding times. Physiology and Behavior, 48, 523-529. doi:10.1016/0031-9384(90)90294-E

Carr, J. A. R., \& Wilkie, D. M. (1997a). Ordinal, phase, and interval timing. In C. M. Bradshaw \& E. Szabadi (Eds.), Time and behavior: Psychological and neurobiological analyses (pp. 265-327). Amsterdam, The Netherlands: Elsevier.

Carr, J. A. R., \& Wilkie, D. M. (1997b). Rats use an ordinal timer in a daily time-place learning task. Journal of Experimental Psychology: Animal Behavior Processes, 23, 232-247.

Daan, S., \& Koene, P. (1981). On the timing of foraging flights by oystercatchers, Haematopus ostralegus, on tidal mudflats. Netherlands Journal of Sea Research, 15, 1-22. doi:10.1016/0077-7579(81)90002-8

Deibel, S. H., Ingram, M. L., Lehr, A. B., Martin, H. C., Skinner, D. M., Martin, G. M., \& Thorpe, C. M. (2014). In a daily time-place learning task, time is only used as a discriminative stimulus if each daily session is associated with a distinct spatial location. Learning \& Behavior, 42, 246-255. doi:10.3758/s13420-014-0142-1

Deibel, S. H., \& Thorpe, C. M. (2013). The effects of response cost and species-typical behaviors on a daily time-place learning task. Learning \& Behavior, 41, 42-53. doi:10.3758/s13420-012-0076-4

Gallistel, C. R. (1990). The organization of learning. Cambridge, MA: MIT Press.

Lukoyanov, N. V., Pereira, P. A., Mesquita, R. M., \& Andrade, J. P. (2002). Restricted feeding facilitates time-place learning in adult rats. Behavioural Brain Research, 134, 283-290. doi:10.1016/S0166-4328(02)00036-0 
Means, L. W., Arolfo, M. P., Ginn, S. R., Pence, J. D., \& Watson, N. P. (2000). Rats more readily acquire a time-of-day go no-go discrimination than a time-of-day choice discrimination. Behavioural Processes, 52, 11-20. doi:10.1016/S0376-6357(00)00109-1

Means, L. W., Ginn, S. R., Arolfo, M. P., \& Pence, J. D. (2000). Breakfast in the nook and dinner in the dining room: Timeof-day discrimination in rats. Behavioural Processes, 49, 2133. doi:10.1016/S0376-6357(00)00068-1

Mistlberger, R. E., De Groot, M. H. M., Bossert, J. M., \& Marchant, E. G. (1996). Discrimination of circadian phase in intact and suprachiasmatic nuclei-ablated rats. Brain Research, 739, 12-18. doi:10.1016/S0006-8993(96)00466-0

Pizzo, M. J., \& Crystal, J. D. (2002). Representation of time in time-place learning. Animal Learning \& Behavior, 30, $387-$ 393. doi:10.3758/BF03195963

Pizzo, M. J., \& Crystal, J. D. (2004a). Evidence for an alternation strategy in time-place learning. Behavioural Processes, 67, 533-537. doi:10.1016/j.beproc.2004.06.004

Pizzo, M. J., \& Crystal, J. D. (2004b). Time-place learning in the eight-arm radial maze. Learning \& Behavior, 32, 240-255. doi:10.3758/BF03196025

Pizzo, M. J., \& Crystal, J. D. (2006). The influence of temporal spacing on time-place discrimination. Learning \& Behavior, 34, 131-143. doi:10.3758/BF03193189
Saksida, L. M., \& Wilkie, D. M. (1994). Time-of-day discrimination by pigeons, Columba livia. Animal Learning and Behaviour, 22, 143-154.

Thorpe, C. M., Bates, M. E., \& Wilkie, D. M. (2003). Rats have trouble associating all three parts of the time-place-event memory code. Behavioural Processes, 63, 95-110. doi:10.1016/S0376-6357(03)00051-2

Thorpe, C. M., \& Wilkie, D. M. (2007). Rats acquire a low-response-cost daily time-place task with differential amounts of food. Learning \& Behavior, 35, 71-78. doi:10.3758/BF03196076

Van der Zee, E. A., Havekes, R., Barf, R. P., Hut, R. A., Nijholt, I. M., Jacobs, E. H., \& Gerkema, M. P. (2008). Circadian time-place learning in mice depends on cry genes. Current Biology, 18, 844-848. doi:10.1016/j.cub.2008.04.077

Widman, D. R., Gordon, D., \& Timberlake, W. (2000). Response cost and time-place discrimination by rats in maze tasks. Animal Learning \& Behavior, 28, 298-309. doi:10.3758/BF03200263

Widman, D. R., Sermania, C. M., \& Genismore, K. E. (2004). Evidence for time-place learning in the Morris water maze without food restriction but with increased response cost. Behavioural Processes, 67, 183-193. doi:10.1016/j.beproc.2004.04.001

Wilkie, D. M., Carr, J. A. R., Siegenthaler, A., Lenger, B., Liu, M., \& Kwok, M. (1996). Field observations of time-place behaviour in scavenging birds. Behavioural Processes, 38, 77-88. 